\title{
High-power photoreceivers for large dynamic range: high frequency photonic RF links
}

H. Meier, F. Schaub, V. Liverini, E. Portuondo-Campa, S. Lecomte

H. Meier, F. Schaub, V. Liverini, E. Portuondo-Campa, S. Lecomte, "Highpower photoreceivers for large dynamic range: high frequency photonic RF links," Proc. SPIE 11852, International Conference on Space Optics - ICSO 2020, 1185245 (11 June 2021); doi: 10.1117/12.2599640

SPIE Event: International Conference on Space Optics - ICSO 2021, 2021, Online Only 


\section{International Conference on Space Optics-ICSO 2020}

Virtual Conference

30 March-2 April 2021

Edited by Bruno Cugny, Zoran Sodnik, and Nikos Karafolas
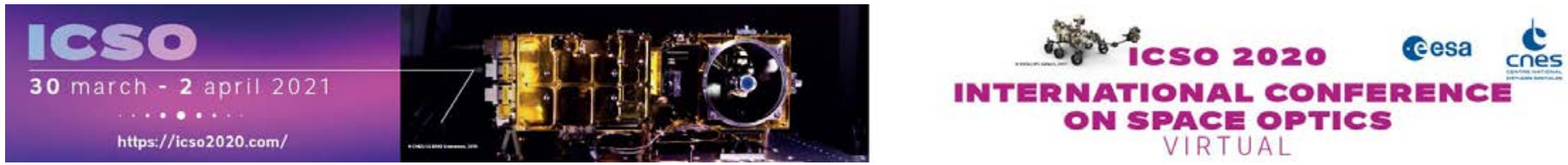

\section{High-power photoreceivers for large dynamic range: high frequency photonic RF links}

\section{Cesa issopreasings denes}




\title{
High-power photoreceiver for large dynamic range - high frequency photonic RF links
}

\author{
F. Schaub ${ }^{\mathrm{a}}$, V. Liverini ${ }^{\mathrm{a}}$, E. Portuondo-Campa ${ }^{\mathrm{b}}, \mathrm{S}$. Lecomte $^{\mathrm{b}}$, H. Meier*a \\ ${ }^{a}$ Albis Optoelectronics AG, Moosstrasse 2a, 8803 Rueschlikon, Switzerland, \\ ${ }^{b}$ Centre Suisse d'Electronique et de Microtechnique (CSEM), Jaquet-Droz 1, 2000 Neuchâtel, \\ Switzerland
}

\begin{abstract}
This contribution summarizes the status of our current development of a novel, high-power and high-linearity photodiode. This photodiode is specifically designed to operate over an exceptionally wide optical input power range from below $-10 \mathrm{dBm}$ up to $+20 \mathrm{dBm}$ without relying on self-biasing effects, making it a versatile solution in many potential analog optical RF applications. The backside illuminated photodiode offers a large active diameter, which enables high coupling efficiency, allows optimization of the optical launch conditions to improve the optical saturation current and linearity as well as to improve the thermal power dissipation, which is required for high power operation. The high-power photodiodes, flip-chip-bonded to their heat-dissipation carriers, have been integrated in a hermetically sealed, rugged package with builtin matching circuit and bias-T. The thermal design and the opto-electrical characterization such as bandwidth and linearity will be presented. Preliminary gamma and proton irradiation testing, have been performed with promising results.
\end{abstract}

Keywords: photonic payload, photodiode, detector, linearity, RF, proton irradiation, gamma irradiation

\section{INTRODUCTION}

Photonic enabled RF satellite payloads demonstrate superior performance, improved size, weight and power (SWaP) and lower susceptibility to EMI compared to legacy, copper-based solutions. In the recent years photonic technologies have been widely used in optical Radio over Fiber (RoF) applications, in backhaul links for mobile telecommunication networks, distribution over low-loss fiber optical links to remote antenna system (satellite base stations, phased array antennas) or microwave signal generation. A main component in any microwave photonic application is a high-linearity, high-power-handling photodiode converting an optical into an electrical RF signal. An important figure of merit for high-power photodiodes performance is the linearity of the photodetector response which allows an analog optical link to operate with high spurious free dynamic range third order (SFDR3) and hence high signal integrity ${ }^{1}$. The available RF output power is the result of the capability of the photodiode to operate at the desired frequency with high average photocurrent and hence drive a large RF signal amplitude across the load which directly improves the noise figure of the analog optical link. The linear operation of a high-speed PIN photodiode is typically limited to the order of $+10 \mathrm{dBm}$ to $+13 \mathrm{dBm}$ optical input power, which limits the available RF power at the output. In comparison, Ka-band uni-travelingcarrier (UTC) or modified UTC (MUTC) photodiodes can handle optical input power in excess of $+20 \mathrm{dBm}^{2}$. However, such very high-power devices often require a high optical input power to operate since they rely on a self-biasing effects to perform at optimal bandwidth ${ }^{3,4,5}$. In this work we have developed a photodiode which exceeds the power handling capabilities compared to a standard PIN photodiode and at the same time does not depend on any self-biasing effect to achieve optimal performance.

An important aspect of operating optical components in spaceborne applications is their capability to resist radiation induced degradation. In the second part of this work, preliminary gamma irradiation and proton irradiation results for the high-power packaged photodiode are presented.

This contribution describes results of the project HOPP, funded by ARTES 5.1 activity 5C.237.

* hektor.meier@albisopto.com; www.albisopto.com 


\section{DESIGN CONSIDERATIONS}

In general, available RF output power, dynamic range and linearity of a photodiode are limited by space charge effects, voltage swing across any series resistance and thermal heating limitations ${ }^{6,7}$.

Under high optical input power condition, the band profile is modified because photo-generated carriers start to accumulate within the junction and hence the high density of charge carriers may compensate the applied electric field. This results in reduced carrier mobility and carrier velocity and thereby reduce the speed of response which may impact the RF output amplitude. Furthermore, the modulation of the electric field and charge velocity potentially degrades the linearity of the detector.

These effects are amplified by high optical power density, a modulation of the electric field as a result of any voltage swing caused by a series resistor at high photo current, as well as increased temperature which degrades the carrier mobility at low electric fields.

Increasing the bias voltage may improve linearity of the photodiode response because it leads to a higher electric field in the junction, making carrier transport less susceptible to above mentioned effects. However, the potential improvement is limited by the increased self-heating and device temperature due to the higher bias voltage. Ultimately, the excess temperature in the junction may result in local melting and catastrophic failure of the detector.

Therefore, the primary design goal is thermal optimization in order to improve high RF output power and linearity as well as reliability and damage threshold. This is achieved by maximizing the active area diameter and by flip-chip bonding the chip on a high thermal conductivity AlN ceramic carrier which conducts generated heat away from the junction.

\section{MANUFACTURING}

The InGaAs/InP high power photodiode chip has been fabricated in the Albis Optoelectronics clean room facility in Rüschlikon, Switzerland. The photodiode is flip-chip mounted on an AlN ceramic carrier using a eutectic AuSn soldering process. Figure 1 shows the backside of the high-power photodiode chip-on-carrier (CoC) with ground-signalground (GSG) pad layout (Albis Optoelectronics product code HPS20Y1-3A). The chip light entry features an antireflective coating which reduces back-reflection of the surface to less than $1 \%$ at a wavelength of $1550 \mathrm{~nm}$. The photodiode diameter is optimized with regards to the trade-off between thermal and optical coupling, device capacitance as well as device bandwidth.

The photodiode has been assembled into a hermetically sealed, rugged package (see Figure 1). The packaged high-power photodiode implements two types of RF matching circuit, a broadband $50 \Omega$ matching (Albis Optoelectronics product code PQW20B) and a narrowband matching optimized at $19 \mathrm{GHz}$ (Albis Optoelectronics product code PQW20N). Both packages offer a single-mode fiber pigtail with FC/APC connector for the optical input, a K-connector for the RF output signal and an internal bias-T.
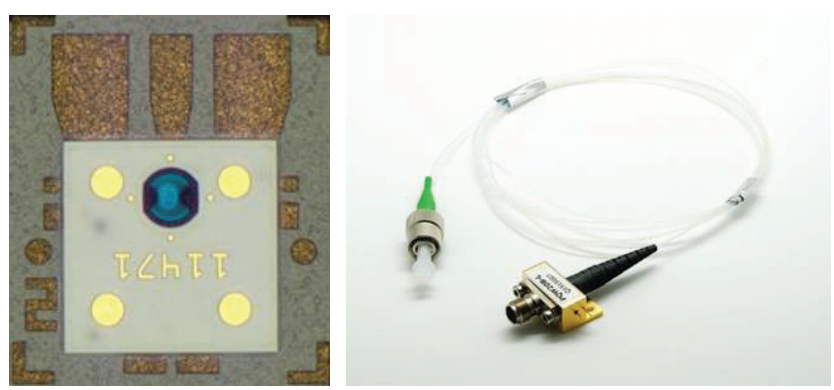

Figure 1 Left: High-power photodiode chip flip-chip mounted on AlN carrier with GSG pad layout. The footprint of the $\mathrm{CoC}$ is $600 \mu \mathrm{m} \times 700 \mu \mathrm{m}$. Right: Hermetically packaged high-power photodiode module. 


\section{PERFORMANCE TEST RESULTS - CHIP-ON-CARRIER}

\subsection{Bandwidth measurements}

The bandwidth measurements of the CoC have been performed using an Agilent 86032A light wave component analyzer. The CoC is contacted using an MPI 67 GHz Titan RF Probe needle and is biased through an Agilent 11612N bias-T. The average optical power is adjusted using an Agilent N7752A optical attenuator. The photodiodes frequency response has a lowpass characteristic with a $3 \mathrm{~dB}$ bandwidth of approximately $21.4 \mathrm{GHz}$ at low optical input power of $-10 \mathrm{dBm}$ and reverse bias voltage of $5 \mathrm{~V}$ (see Figure 2). This demonstrates the capability of the photodiode chip to operate at low optical input power without relying on self-biasing effects. The measurement at $95^{\circ} \mathrm{C}$ show no degradation in bandwidth, which means, that temperature alone is not affecting the carrier transport as long as sufficient large electric field and no optical saturation effect is present.
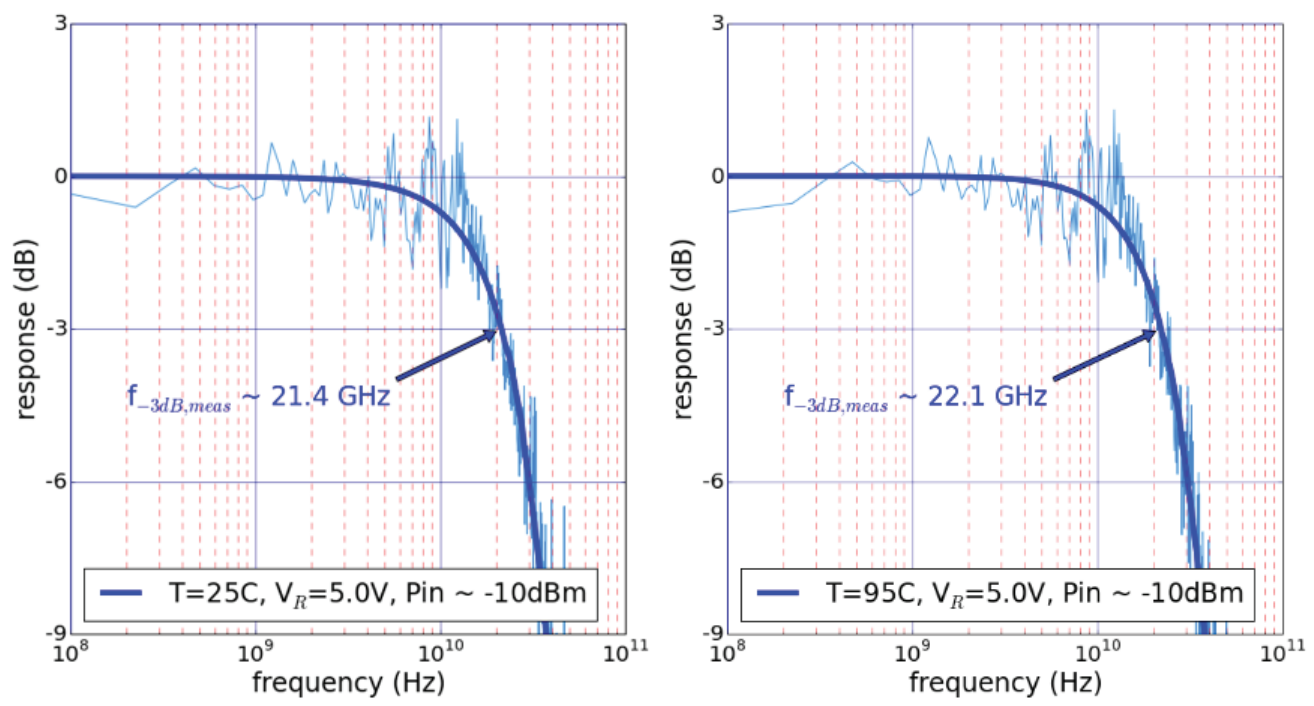

Figure 2 Frequency response of $\mathrm{CoC}$ at $-10 \mathrm{dBm}$ optical input power and $5 \mathrm{~V}$ reverse bias voltage, Left: at $25^{\circ} \mathrm{C}$, Right: at $95^{\circ} \mathrm{C}$.

\subsection{Optical saturation current measurements}

Linearity is an ambiguous term. In the context of this work, we are determining the linear regime of the photodiode by measuring the $1 \mathrm{~dB}$ compression point. This is determined as a function of the RF output vs. the RF input power where the RF output power at the fundamental frequency decreases by $1 \mathrm{~dB}$ or $10 \%$, respectively from its linear relationship with respect to the RF input power. At this point the device goes into optical saturation and generates increased higher order harmonics or non-linearities. The optical RF input power is a function of its extension ratio (ER) and is proportional to the average optical input power which is itself proportional to the average photocurrent generated by the photodiode. Therefore, for a fixed ER, it is common to describe the linearity of the photodiode by the average photocurrent at the $1 \mathrm{~dB}$ compression point. This is called the optical saturation current.

The optical saturation current measurements (see Figure 3) are obtained using a Keysight E8361A PNA, an iXblue $70 \mathrm{GHz} 1550 \mathrm{~nm}$ VNA Modbox with ER 14 dB, an IPG Photonics EAD-500-C EDFA and an Agilent N7752A optical attenuator for average optical power measurements. The $\mathrm{CoC}$ is mounted on a RF mount and biased through the internal bias-T of the PNA. The bias voltage is carefully adjusted to guarantee the voltage at the anode and cathode of the CoC. The sample is mounted on a 3D motorized stage allowing accurate optical alignment with a standard single mode fiber (SMF), see Figure 4. The measurement is done at different distances (d) between the light entry of the CoC and the tip of 
the SMF. By increasing the distance between the fiber and the chip, the optical power density on the detector is decreased. The results are obtained at room temperature without any active cooling of the device.

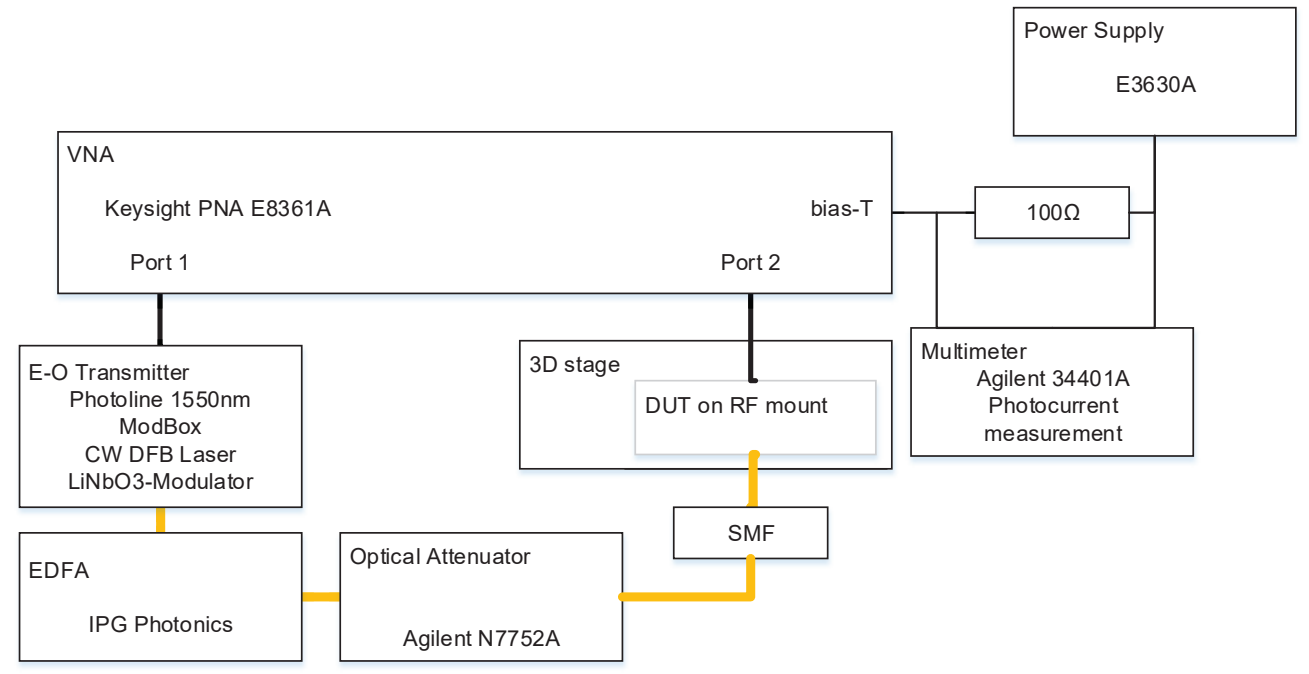

Figure 3 Experimental setup for RF measurement of CoC on 3D motorized stage.

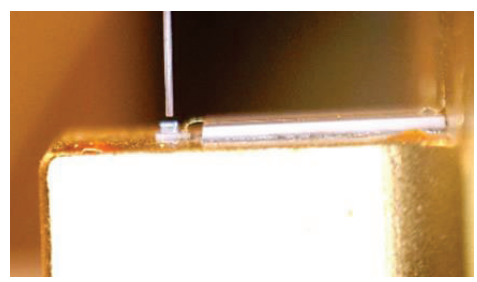

Figure 4 SMF alignment on the light entry of the CoC using 3D motorized stage.

The relative RF output power at $20 \mathrm{GHz}$ as function of average photo-current and at different distances of the SMF from the backside of the photodiode chip is shown in Figure 5. The results show that the optical power density on the active area impacts the optical saturation current. At $150 \mu \mathrm{m}$ distance between the SMF tip and the backside of the photodiode chip, the optical saturation current of the photodiode is $70 \mathrm{~mA}$ at $8 \mathrm{~V}$. This corresponds to an average optical input power of $+20.8 \mathrm{dBm}$. Furthermore, no significant degradation of coupling efficiency has been detected up to a distance of $150 \mu \mathrm{m}$, but we observed an increased responsivity at higher photocurrent which we attribute to the increased device temperature. 


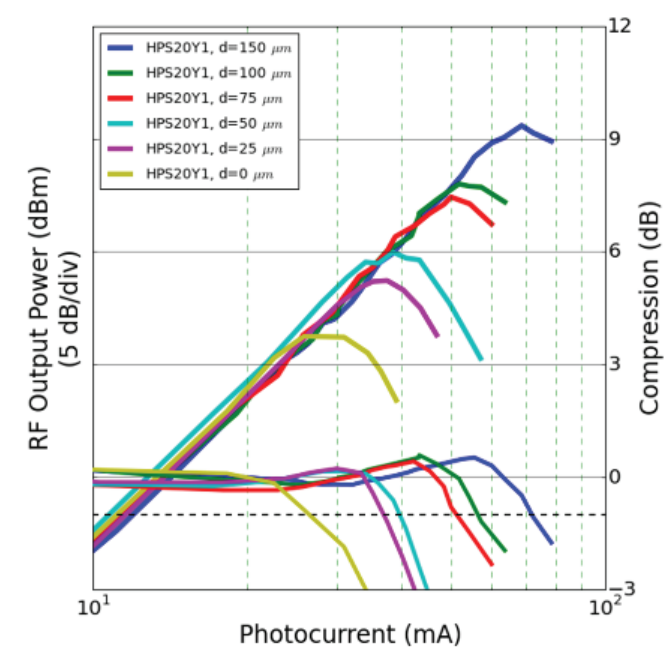

Figure 5 Relative RF output power measurement at $20 \mathrm{GHz}$ and $8 \mathrm{~V}$ reverse bias voltage on the left y-axis and RF power compression on the right $y$-axis as a function of measured average photocurrent for the CoC. The different curves show the behavior of the photodiode as a function of the distance between the optical fiber tip and the backside light entry of the photodiode.

\section{PERFORMANCE TEST RESULTS - PACKAGE PHOTODIODE}

\subsection{Bandwidth measurements}

The bandwidth measurements of the packaged photodiode have been performed as schematically represented in Figure 6 . The optical modulation is provided by the interference of two CW lasers. The wavelength of the first lasers (PPCL550, Pure Photonics) is fixed at $1559 \mathrm{~nm}$ while the wavelength of the second laser (CTL 1550, Toptica Photonics) slowly scans between $1559.0 \mathrm{~nm}$ and $1559.3 \mathrm{~nm}$. In this manner, the interference of the two lasers produces a modulated optical signal (beatnote) at frequencies between 0 and approximately $37 \mathrm{GHz}$.

The lasers are independently amplified by two home-built EDFAs with adjustable pump power and thereafter combined in a polarization maintaining 50/50 fiber combiner. One of the combiner outputs is monitored with an optical power meter (S145C and PM110D, Thorlabs) whereas the other output is connected to a second PM fiber combiner with a $90 / 10$ splitting ratio used to sample $10 \%$ of the power onto an optical spectrum analyzer (OSA, AQ6317B, Ando Electric).

The pump power of the EDFAs is first roughly adjusted to obtain the desired photocurrent in the Device-Under-Test (DUT) and then fine-tuned to ensure that the two signals have the same amplitude, which results in a $100 \%$ modulation depth of the optical signal. This power-balance adjustment is performed by monitoring the signals in the OSA. The electrical spectrum analyzer (N9030A PXA, Keysight) is set to max-hold acquisition mode recording the peak value of the beatnote signal as it sweeps across the desired frequency range. The losses of the cable and the DC-block connected to the RF spectrometer have been measured and compensated for. 


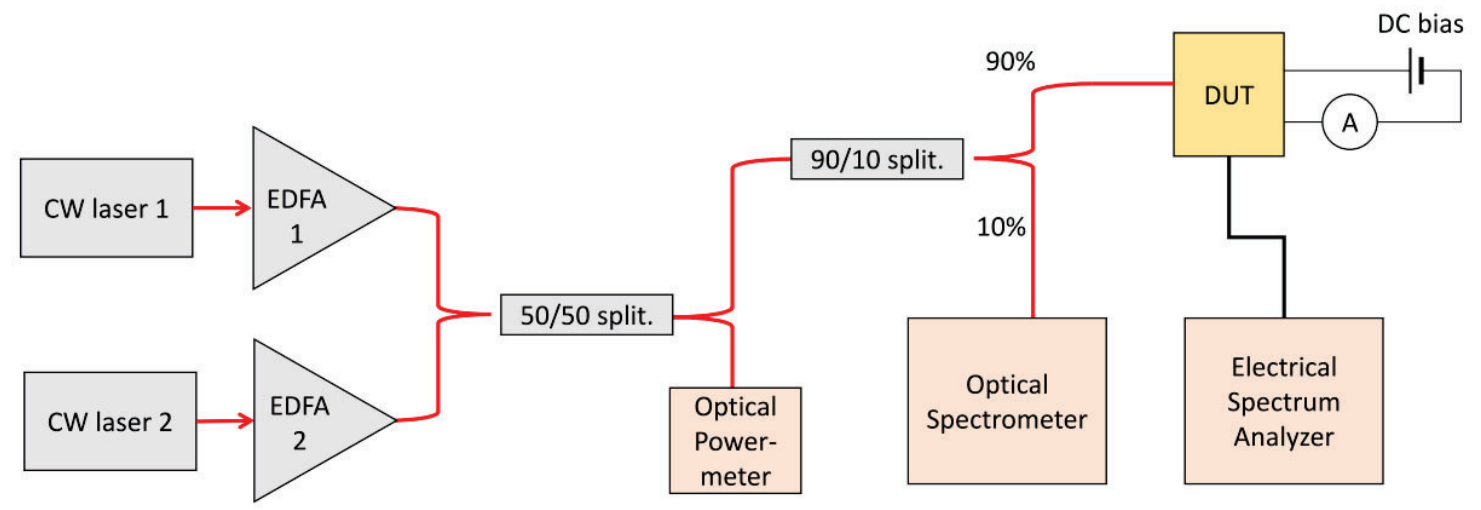

Figure 6 Experimental setup for high-power S12 measurement.

Using this setup, the S12 frequency response of the broadband (PQW20B) and narrowband (PQW20N) packaged photodiode have been measured. The results for the broadband module show a high $3 \mathrm{~dB}$ bandwidth of around $24 \mathrm{GHz}$ over a large range of average photocurrent, see Figure 7. The $3 \mathrm{~dB}$ bandwidth of the PQW20B is slightly higher than for the $\mathrm{CoC}$. The difference can be explained by the internal $50 \Omega$ termination resistor used in the package, which increases the RC time limiting bandwidth of the photodiode. Increasing the bias voltage improves the bandwidth at higher photocurrent as optical saturation effects are suppressed by the higher electric field. At a bias voltage of $10 \mathrm{~V}$ the bandwidth remains above $22 \mathrm{GHz}$ up to $50 \mathrm{~mA}$. The bandwidth decreases at lower photocurrent than we would expect from the $\mathrm{CoC}$ measurement. Despite the fact, that the photodiode within the package is illuminated with a distance of approximately 100-150um between the SMF tip and the backside of the photodiode. For the CoC measurement, the bias is applied through a bias-T which includes a DC block isolating the DC signal from the $50 \Omega$ load in the PNA. In case of the PQW20B, the internal bias-T includes a $10 \mathrm{Ohm}$ series resistor for ESD protection on the biasing pin. In conjunction with the matching, this leads to an internal serial resistance which reduces the bias voltage on the photodiode at higher photo current. As a result the packaged photodiode saturates at significantly lower photocurrent than the $\mathrm{CoC}$.

The frequency response measurements of the narrowband packaged photodiode (PQW20N) show the RF output power peaking at $19 \mathrm{GHz}$ which is the result of the specific matching at this frequency, see Figure 7.
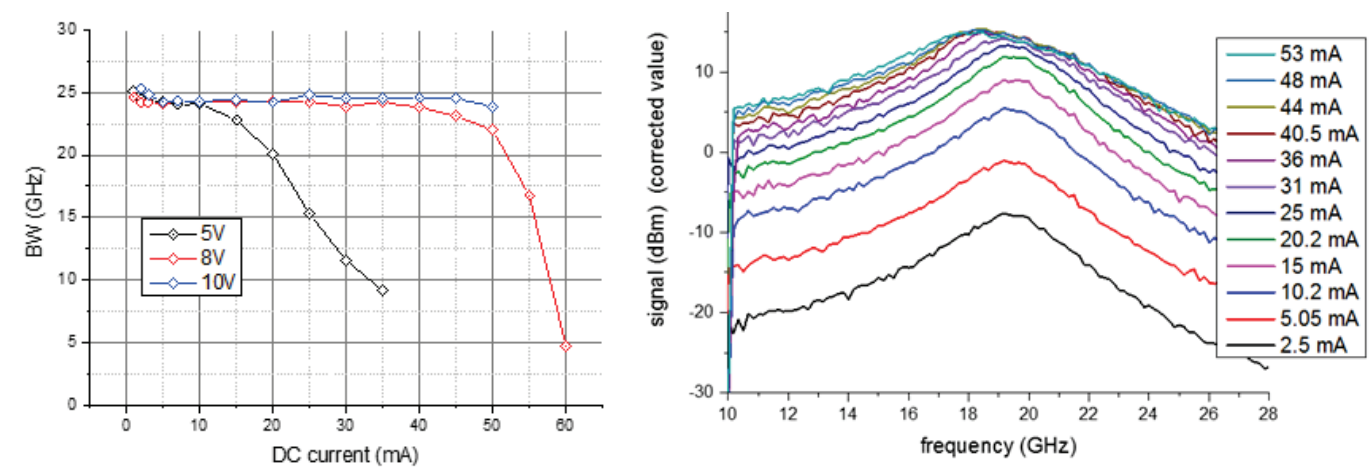

Figure 7 Left: $-3 \mathrm{~dB}$ bandwidth for different photocurrent and reverse bias voltage of $10 \mathrm{~V}$ for a broadband packaged photodiode. Right: Frequency response of the RF output for different optical powers at reverse bias voltage of $10 \mathrm{~V}$ for a narrow band packaged photodiode 


\subsection{Optical saturation current measurements}

For the saturation current measurements of the packaged photodiode the same setup shown in Figure 6 has been used. The RF output power as a function of the photocurrent at $19 \mathrm{GHz}$ (see Figure 8) shows that the narrowband module (PQW20N) offers higher RF output power of up to $+15 \mathrm{dBm}$ compared to $+12 \mathrm{dBm}$ for the broadband module.

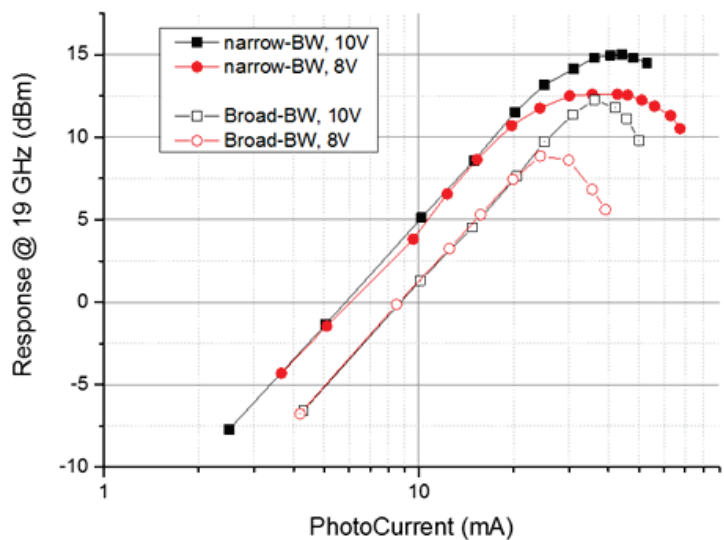

Figure $8 \mathrm{RF}$ output power at $19 \mathrm{GHz}$ as a function of photocurrent and bias voltage for the broadband (PQW20B) and narrowband module (PQW20N).

\section{IRRADIATION TESTING}

\subsection{Gamma irradiation testing}

The gamma irradiation testing has been performed in the ESA-ESTEC Co60 facilities, Nordwijk, Netherlands. The test procedure is shown in Figure 9. The sample consists of four hermetically packaged photodiodes (PQW20B). One additional sample (S/N Q1804006) was stored without being exposed to irradiation and acts as reference sample. Two samples have been biased at $5 \mathrm{~V}$ during irradiation testing while two samples remained unbiased. Before irradiation testing all packaged photodiodes under test have been characterized with respect to responsivity, dark current, electrical return loss $\left(\mathrm{S}_{11}\right)$ and $3 \mathrm{~dB}$ bandwidth $\left(\mathrm{S}_{12}\right)$. The total dose of $106 \mathrm{krad}$ which corresponds to approximately 15 years exposure to geostationary orbit (GEO) has been applied in four runs whereas dark current has been measured after each run at ESTEC. After the last irradiation step, the component has been relaxed for $24 \mathrm{~h}$ before another dark current measurement has been performed at ESTEC. After the samples have been returned to Albis dark current, responsivity and S-parameter have been measured again and thereafter curing at $100^{\circ} \mathrm{C}$ for one week. 


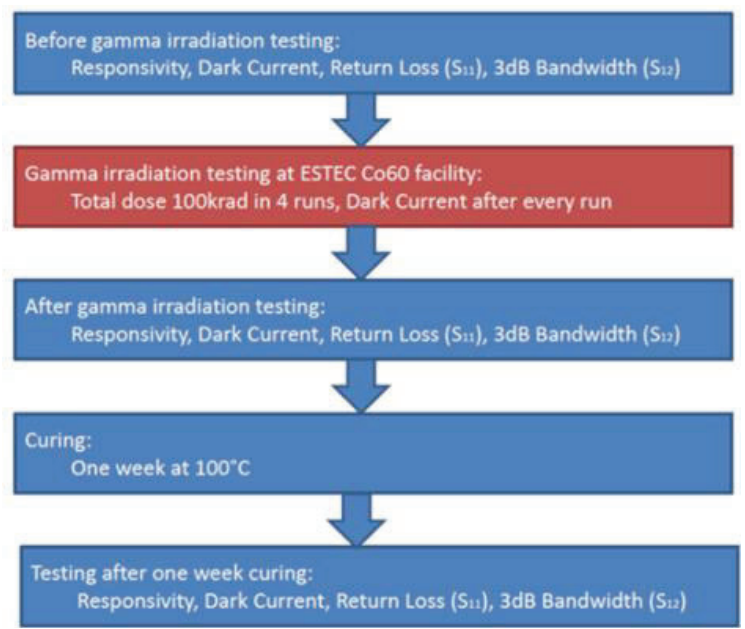

Figure 9 Gamma Irradiation Test Procedure

The dark current values before and after each irradiation step as well as after curing are shown in Figure 10. Additionally, Figure 11 depicts the $-3 \mathrm{~dB}$ bandwidth measurements and responsivity values before, after irradiation and after curing. The dark current, responsivity as well as the S-parameter measurements do not show any degradation compared to the initial measurement.

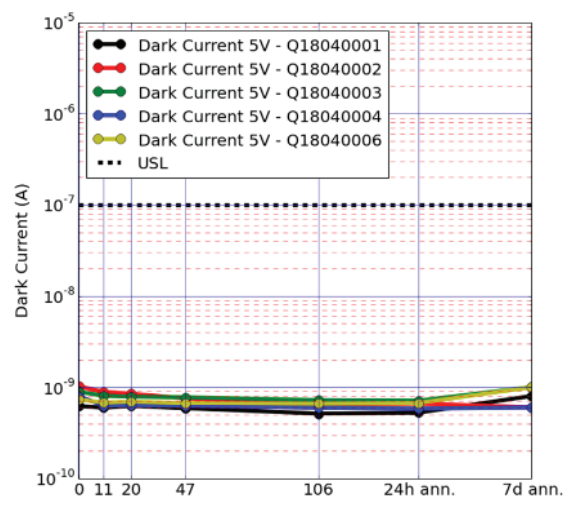

Figure 10 Dark current at room temperature and 5V bias voltage as a function of Gamma irradiation dose in krad, 24h and $7 \mathrm{~d}$ curing. USL stands for upper specification limit of the photodiode. 

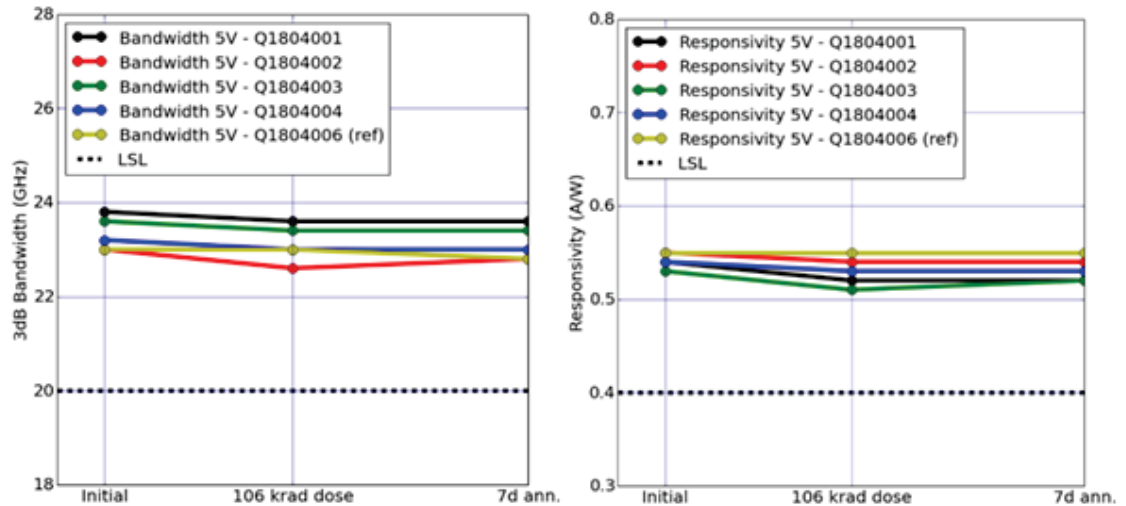

Figure 11 Responsivity at wavelength of $1550 \mathrm{~nm}$ and bias voltage of $5 \mathrm{~V}$ before and after irradiation as well as after one week curing at $100^{\circ} \mathrm{C}$. LSL stands for lower specification limit for the packaged photodiodes.

The results of the gamma irradiation testing are summarized in Table 1. None of the tested devices shows significant degradation.

Table 1 Gamma irradiation result summary

\begin{tabular}{|c|c|c|c|c|}
\hline Photodiode S/N & $\begin{array}{c}\text { Reverse Bias } \\
(\mathbf{V})\end{array}$ & $\begin{array}{c}\text { Total Dose } \\
\text { (krad) }\end{array}$ & $\begin{array}{c}\text { Equiv. GEO } \\
\text { mission time (y) }\end{array}$ & Pass / Fail \\
\hline Q1804001 & 5 & 106 & $\sim 15$ & Pass \\
\hline Q1804002 & 5 & 106 & $\sim 15$ & Pass \\
\hline Q1804003 & Unbiased & 106 & $\sim 15$ & Pass \\
\hline Q1804004 & Unbiased & 106 & $\sim 15$ & Pass \\
\hline
\end{tabular}

\subsection{Proton irradiation testing}

The proton irradiation testing was conducted at the Proton Irradiation Facility (PIF) of the Paul-Scherrer Institute (PSI), Villingen, Switzerland. The test plan is depicted in Figure 12. Before irradiation testing, all packaged photodiodes have been tested with regards to dark current, responsivity and electrical return loss $\left(\mathrm{S}_{11}\right)$ and $3 \mathrm{~dB}$ bandwidth $\left(\mathrm{S}_{12}\right)$.

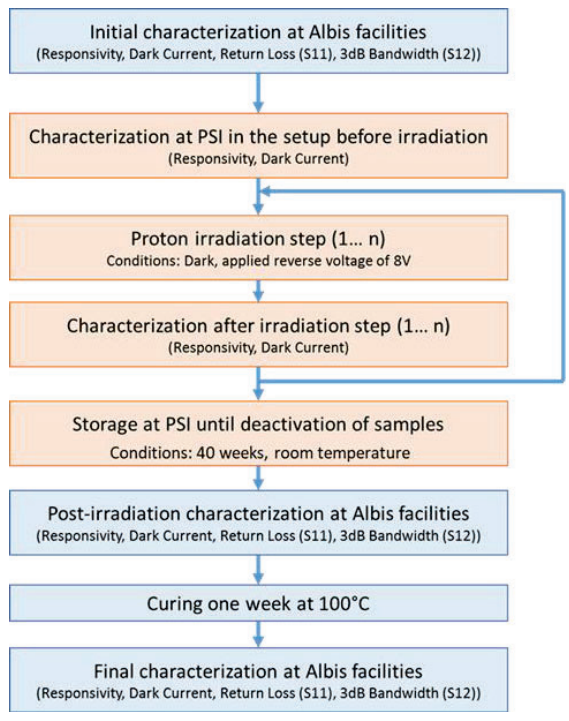

Figure 12 Proton irradiation test procedure. 
Intermediate measurements of S-parameters and thereby assess a potential change in capacitance of the device have not been possible. Therefore, it was decided to expose the sample to different levels of fluence and verify the S-parameters in post-irradiation measurements. Two modules have been exposed to moderate irradiation while two packaged photodiodes have been exposed to radiation largely exceeding any reasonable space mission in LEO. An overview of the applied dose is given in Table 2. The equivalent LEO mission time is estimated based on an averaged proton flux of approximately $1 \mathrm{e} 2$ protons $/\left(\mathrm{cm}^{2} \mathrm{~s}\right)$ for ISS low-earth-orbit (LEO), condition of solar maximum and no shielding ${ }^{8}$.

Due to the limited proton beam width $(5 \mathrm{~cm})$, only two modules have been simultaneously exposed (see Figure 13).

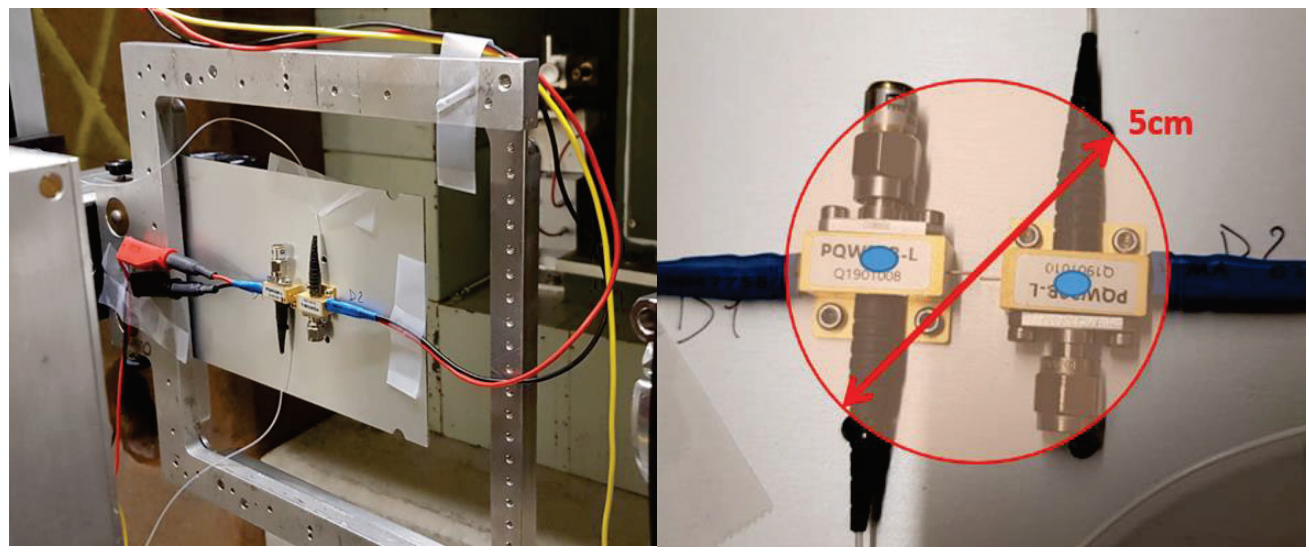

Figure 13 Two packaged photodiodes mounted in the proton beam line at PSI.

During proton irradiation the dark current increases as a function of the accumulated dose (see Figure 14). The dark current does not reach the upper specification limit of $100 \mathrm{nA}$. Considering the main applications for the high-power packaged photodiode are operation at high optical input power thus an increase of the dark current to around $100 \mathrm{nA}$ is not expected to affect the overall system performance.

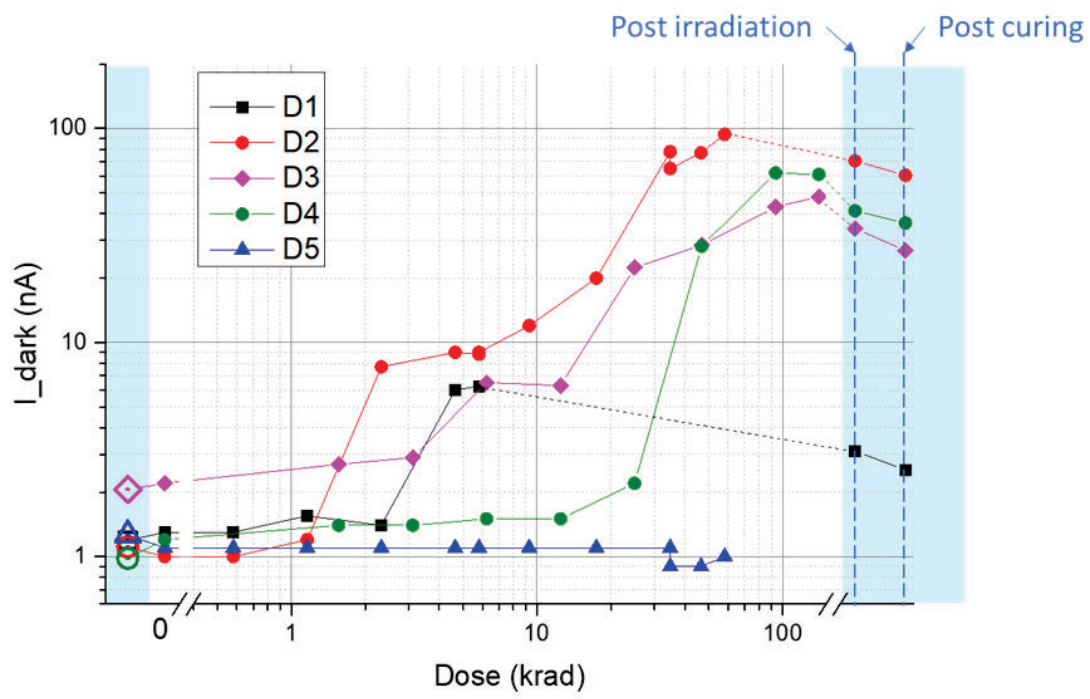

Figure 14 Room temperature dark current at $5 \mathrm{~V}$ reverse bias voltage, before and directly after proton irradiation, as well as after 40 weeks storage and followed by one week curing at $100^{\circ} \mathrm{C}$., D1: S/N Q1901001, D2: S/N Q1901003, D3: S/N Q1901008, D4: S/N 1901010, D5: reference sample without irradiation.

The $3 \mathrm{~dB}$ bandwidth and responsivity (see Figure 15) do not show significant alteration. Same applies for the electrical return loss $\left(\mathrm{S}_{11}\right)$. 

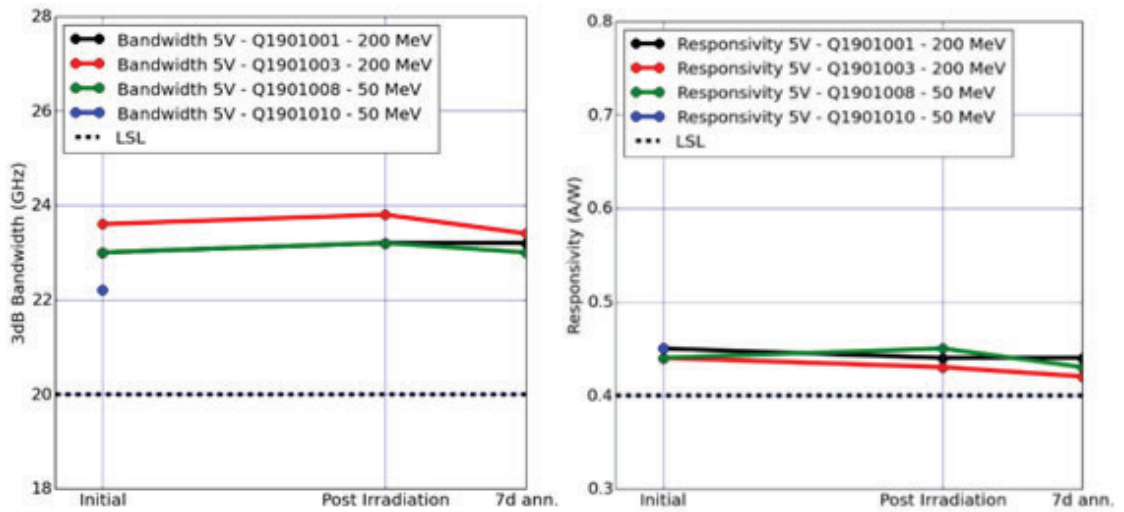

Figure $15-3 \mathrm{~dB}$ bandwidth measurement and responsivity at wavelength of $1550 \mathrm{~nm}$ and $5 \mathrm{~V}$ bias voltage before proton irradiation testing, after irradiation and 40 weeks relaxation as well as one week curing at $100^{\circ} \mathrm{C}$.

The results of the proton irradiation testing are summarized in Table 2. Due to a fiber breakage as result of handling error, responsivity and $3 \mathrm{~dB}$ bandwidth measurement of module Q1901010 could not be performed.

Table 2 Proton Irradiation Test Results.

\begin{tabular}{|c|c|c|c|c|c|c|c|}
\hline $\begin{array}{c}\text { Photodiode } \\
\text { S/N }\end{array}$ & Reference & $\begin{array}{c}\text { Reverse Bias } \\
(\mathbf{V})\end{array}$ & $\begin{array}{c}\text { Cumulated } \\
\text { Fluence } \\
\left(\mathbf{p} / \mathbf{c m}^{2}\right)\end{array}$ & $\begin{array}{c}\text { Total } \\
\text { Dose } \\
(\mathbf{k r a d})\end{array}$ & $\begin{array}{c}\text { Proton } \\
\text { Energy } \\
\mathbf{( M e V )}\end{array}$ & $\begin{array}{c}\text { Equiv. } \\
\text { LEO } \\
\text { mission } \\
\text { time (y) }\end{array}$ & $\begin{array}{c}\text { Pass / } \\
\text { Fail }\end{array}$ \\
\hline Q1901001 & D1 & 8 & $1 \mathrm{e} 11$ & 5.8 & 200 & $\sim 32$ & Pass \\
\hline Q1901003 & D2 & 8 & $1 \mathrm{e} 12$ & 58 & 200 & $\sim 317$ & Pass \\
\hline Q1901008 & D3 & 8 & $9 \mathrm{e} 11$ & 140 & 50.8 & $\sim 285$ & Pass \\
\hline Q1901010 & D4 & 8 & $9 \mathrm{e} 11$ & 140 & 50.8 & $\sim 285$ & - \\
\hline
\end{tabular}

\section{CONCLUSION}

Performance and preliminary environmental test results of a novel high-power photodiode, optimized for a high dynamic range of $-10 \mathrm{dBm}$ up to $+20 \mathrm{dBm}$ optical input power have been presented. The effect of optical illumination, biasing and series resistance on high-power bandwidth and linearity have been demonstrated. The photodiode chip provides linear RF response at $20 \mathrm{GHz}$ up to $70 \mathrm{~mA}$ photocurrent at $8 \mathrm{~V}$ without active cooling. Gamma irradiation testing show no effect on the packaged photodiode and proton irradiation testing increases dark current. However, at no point a dark current reaches a level which would be critical for operation of the photodiode.

Acknowledgment: We thank the ESA technical office Bruno Leone and the team at ESTEC as well as PSI for the facility support.

Research funding: This work has been supported by ESA ARTES 5.1 activity 5C.237.

Conflict of interest: The authors declare no conflicts of interest regarding this contribution. 


\section{REFERENCES}

[1] Urick, V. J., McKinney, J. D. and Williams, K. J., [Fundamental of microwave photonics], John Wiley \& Sons, Inc., (2015).

[2] Li, Z., Pan, H., Chen H., Beling A. and Campbell, J.C., "High-Saturation-Current Modified Uni-Traveling-Carrier Photodiode With Cliff Layer," IEEE Journal of Quantum Electronics,46(5), 626-632 (2010).

[3] Ito, H., Kodama, S., Muramoto, Y., Furuta, T., Nagatsuma, T. and Ishibashi, T., "High-Speed and High-Output InPInGaAs Unitraveling-Carrier Photodiodes," IEEE Journal of Selected Topics in Quantum Electronics, 10(4), 709-727 (2004).

[4] Rouvalis, E., Baynes, F. N., Xie X., Li K., Zhou Q., Quinlan F., Fortier, T., Diddams, S.A., Steffan A., Beling, A. and Campbell, J., "High-Power and High-Linearity Photodetector Modules for Microwave Photonic Application," Journal of Lightwave Technology, 32(20), 3810-3816 (2014).

[5] Chtioui, M., Lelarge, F., Enard, A., Pommereau, F., Carpentier, D., Marceaux, A., Van Dijk, F. and Achouche, M., "High Responsivity and High Power UTC and MUTC GaInAs-InP Photodiodes," IEEE Photonics Technology Letters, 24(4), 318-319 (2012).

[6] Chtioui, M., Enard, A., Carpentier, D., Lelarge, F., Rousseau, B., Achouche, M., Marceaux, A., Renoult, A., Feuillet, C., Queguiner, M. and Merlet, T., "High Power UTC Photodiodes Design and Applications for Analog Fiber Optics Links," International Topical Meeting on Microwave Photonics, (2009).

[7] Williams, K. and Esman, R., "Design Considerations for High-Current Photodetectors," Journal of Lightwave Technology, 17(8), 1443-1454 (1999).

[8] Martines, L.M.S., [Analysis of LEO Radiation Environment and its Effects on Spacecraft's Critical Electronic Devices], Dissertations and Theses, 102, Embry-Riddle Aeronautical University, (2011). 